\title{
REFLEXÕES SOBRE O ENSINO DE ESTATÍSTICA NA EDUCAÇÃO BÁSICA: LIÇÕES QUE PODEM SER APRENDIDAS A PARTIR DA FEIRA DE CIÊNCIAS E MATEMÁTICA DA BAHIA - FECIBA
}

\author{
Irene Mauricio Cazorla, Kátia Lima de Sousa Ramos e Rogério Lima de Jesus \\ Universidade Estadual de Santa Cruz, UESC, Brasil \\ Instituto Anísio Teixeira, IAT, Brasil \\ icazorla@uol.com.br
}

A importância do ensino de Estatística na Educação Básica fez com que o Brasil recomendasse sua inclusão no currículo (Brasil, 1997, 1998, 2002) e a comunidade acadêmica e escolar tem-se debruçado, tanto para investigar os diversos fenômenos do ensino e da aprendizagem estatística, quanto de sua institucionalização nas escolas, onde os professores utilizam a Estatística em projetos que envolvem levantamento, sistematização e apresentação em eventos da comunidade escolar. Este trabalho analisa os projetos vencedores de quatro edições da Feira de Ciências e Matemática da Bahia - Feciba e tece reflexões sobre o papel potencial do seu ensino, pois essa transcende as barreiras da disciplinaridade e do conteudismo, sistematiza o pensamento científico, possibilitando a leitura do mundo não apenas dos fenômenos da natureza, mas, especialmente, os sociais, tão presentes na sociedade, em um momento em que o Brasil discute a Base Nacional comum para o Ensino Médio e para as Licenciaturas.

\section{INTRODUÇÃO}

Atualmente, o Brasil está revendo sua política educacional, uma vez que apesar do alto investimento em políticas e programas educacionais nos últimos 30 anos, como a publicação da Lei de Diretrizes e Bases da Educação - LDB (Brasil, 1996), dos Parâmetros Curriculares Nacionais PCN (Brasil, 1997, 1998, 2002), da Política Nacional de Formação de Profissionais do Magistério da Educação Básica (Brasil, 2009), dentre outros, os indicadores educacionais que medem o desempenho dos estudantes, sinalizam uma desaceleração na aprendizagem e no desenvolvimento de competências e habilidades traçadas como meta pelos Planos de Educação.

O Plano Nacional de Educação 2014-2024 (Brasil, 2014a), que envolve os Planos Estaduais e Municipais, tem metas ousadas para o próximo decênio. Contudo, os desafios são enormes, pois se reconhece que o fenômeno educacional, em especial, o da escola pública. Além disso, a maioria dos educadores concorda que o modelo atual de formação de professores e da organização curricular na escola, pautada na disciplinaridade e na fragmentação do conhecimento, não dá conta dos desafios de uma sociedade, fortemente marcada pela tecnologia e mudança radical de valores, onde os estudantes não são mais agentes passivos no processo ensino aprendizagem.

Nesse contexto, os gestores da política educacional, comunidade escolar e comunidade acadêmica vêm estudando a reformulação da matriz curricular tanto do ensino médio, quanto dos cursos de Licenciaturas e de formação continuada de professores da Educação Básica, a partir da Base Nacional Comum da Educação (Brasil, 2014b), em especial para o Ensino Médio, onde o foco da discussão continua a estar centrado em palavras chaves tais como: interdisciplinaridade, transversalidade, colaboração e formação para autonomia.

Este artigo tece reflexões de como o ensino de Estatística na Educação Básica pode ser um grande aliado na consecução de um novo modelo de ensino e de formação de professores, a partir da experiência da política de Educação Científica na escola pública, realizada pela Secretaria da Educação do Estado da Bahia, em especial, o da Feira de Ciências e Matemática da Bahia - Feciba.

\section{O ENSINO DE ESTATÍSTICA NA EDUCAÇÃO BÁSICA}

No componente curricular Matemática, segundo os PCN, os conteúdos de Estatística para o Ensino Fundamental, estão incluídos em um dos quatro blocos, denominado "Tratamento da Informação" (Brasil, 1997, 1998) e, no Ensino Médio (Brasil, 2002), em um dos três eixos, denominado "Análise de Dados". Os conteúdos conceituais e procedimentais de Estatística recomendados vão desde a leitura de informações contidas em tabelas, gráficos e medidas resumo, até a coleta, organização, construção de tabelas e gráficos, bem como o cálculo de medidas de tendência central e de dispersão.

In: M.A. Sorto (Ed.), Advances in statistics education: developments, experiences and assessments. Proceedings of the Satellite conference of the International Association for Statistical Education (IASE), July 2015, Rio de Janeiro, Brazil. 
Já nos outros componentes curriculares é fortemente estimulada a formação de usuários de Estatística. Por exemplo, nos PCN de Ciências da Natureza se recomenda que ao trabalhar seus conteúdos, os estudantes realizem pesquisas, busquem informações, registrem observações, anotem e quantifiquem dados, utilizando conhecimentos relacionados à Língua Portuguesa, Matemática e de outras áreas, cabendo ao professor a tomada de decisões a respeito de suas intervenções e da maneira como tratará os temas, de forma a propiciar aos estudantes uma abordagem mais significativa e contextualizada (Brasil, 1997, p. 44).

O mesmo é encontrado nas três competências gerais da área de Ciências Humanas e suas Tecnologias. A primeira competência "representação e comunicação" relaciona-se diretamente com capacidade de processar e comunicar informações e conhecimentos de forma ampla, desenvolvendo diferentes habilidades de comunicação (oral, escrita, gráfica, pictórica etc.). A segunda "investigação e compreensão" refere-se aos diferentes procedimentos, métodos, conceitos e conhecimentos que são mobilizados e/ou construídos/reconstruídos nos variados processos de intervenção no real, que são sistematizados a partir da resolução de problemas relacionados às análises acerca da realidade social. E, a terceira "contextualização sociocultural", refere-se à diversidade e, portanto, à constituição dos diferentes significados que saberes de ordem variada podem assumir em diversos contextos sociais. (Brasil, 2002, p. 30).

Para desenvolver essas competências e habilidades, os estudantes devem ser sujeitos ativos na realização das atividades escolares e não meros espectadores passivos do discurso dos docentes e/ou das fontes, o que implica a necessidade de uma prática docente centrada na associação ensinopesquisa, de forma permanente no âmbito das aulas regulares e não apenas nos momentos ocasionais dos chamados "projetos extracurriculares".

Compreender como se propaga uma doença contagiosa como o Ebola ou a Dengue, ou como são realizadas as pesquisas eleitorais e como sua divulgação pode alterar o rumo de um pleito eleitoral, dentre outros fenômenos naturais ou socais, é crucial para a formação humana, científica e cidadã do estudante e essa compreensão precisa sem dúvida dos conhecimentos da Estatística.

\section{O ENSINO DE ESTATÍSTICA NOS CURSOS DE LICENCIATURA}

No modelo atual de formação de professores, cabe a Licenciatura em Matemática a formação dos professores responsáveis pelo ensino dos conteúdos conceituais e procedimentais de Estatística na Educação Básica e, às Licenciaturas de Ciências, Biologia, Geografia e Sociologia cabe a formação de "consumidores" e "produtores" de informações estatísticas.

A matriz curricular da Licenciatura em Matemática, em geral, possui no máximo duas disciplinas de Estatística (Cazorla 2006; Viali, 2008). A título de ilustração, transcrevemos a ementa das disciplinas de uma conceituada universidade estadual paulista. Estatística descritiva: distribuições de frequências; representação gráfica das variáveis quantitativas; medidas de tendência central e dispersão. Probabilidade: definição e propriedades; elementos de Análise Combinatória; probabilidade condicional e independência; Teorema de Bayes. Variáveis Aleatórias: definição, função de distribuição acumulada, esperança de variáveis aleatórias; propriedades. Tipos de variáveis aleatórias, exemplos e relações; momentos, aplicações. Os principais modelos discretos e contínuos: Binomial, Geométrico, Pascal, Hipergeométrico, Poisson. Uniforme, Normal, Exponencial, Gamma, Qui-quadrado. A ementa da segunda disciplina envolve conteúdos que embasa a estimação pontual e por intervalo e teste de hipóteses.

Analisando entre o que deve ser ensinado de Estatística na Educação Básica e a formação do professor de Matemática observa-se a marcada ênfase nos conteúdos e não na didática e no uso da Estatística para a leitura de mundo e a compreensão dos fenômenos naturais e sociais, como preconizado por Batanero (2001), Cazorla \& Castro (2008) e Gal (2002). Além disso, não existem disciplinas que deem uma formação científica tanto para as ciências da natureza, quanto das ciências sociais, que permita a este professor transitar nas diversas áreas de conhecimento que estudam os fenômenos naturais e sociais, que lhe permita fazer parte de equipes interdisciplinares, tornando-se alinhavadores e modeladores quantitativos dos fenômenos que permeiam o mundo e a sociedade. Em outras palavras, os professores de Matemática estão preparados para operacionalizar, do ponto de vista matemático, os conceitos e procedimentos estatísticos, mas não estão aparelhados para utilizá-los no contexto da investigação científica ou social, em nível escolar. 
Analisando os cursos de licenciaturas usuárias e produtoras de Estatística tais como Biologia, Ciências, Geografia e Sociologia, observa-se que, em geral, essas têm uma disciplina de Estatística. Por exemplo, no caso da Biologia tem a Bioestatística ou Estatística Aplicada a Biologia, com ementa: Introdução. Análise exploratória de dados. Probabilidade e avaliação de testes diagnósticos. Distribuições de probabilidades. Inferência estatística: estimação e teste de hipóteses. Correlação e Regressão. Tabelas de contingência. Testes não paramétricos. No caso da Sociologia ou Ciências Sociais: Introduzir conceitos e técnicas básicas da Estatística como instrumentos para o desenvolvimento de pesquisas em ciências sociais. Da definição das questões da pesquisa ao levantamento dos dados. Fontes de dados secundárias e fontes primárias. Tratamento e organização das informações. Distribuição de frequência. Medidas de tendência e de variabilidade. Representação gráfica e tabular. Princípios da amostragem nas ciências sociais. Introdução à probabilidade e suas principais distribuições. Medidas de correlação e associação.

Assim, observa-se que estas licenciaturas, usuárias de Estatística, têm maior aderência à formação para a Educação Básica do que a Licenciatura em Matemática, cujas disciplinas focam apenas os conteúdos. Nesse sentido, cabe a comunidade de educadores estatísticos (Cazorla, Kataoka, Silva, 2010) focar a investigação na formação estatística de professores da Educação Básica e na pesquisa sobre o ensino de Estatística para esse nível de ensino (Cazorla e Santana, 2010).

\section{A FEIRA DE CIÊNCIAS E MATEMÁTICA DA BAHIA - FECIBA}

A Secretaria da Educação do Estado da Bahia implementou, em 2011, a Feira de Ciências e Matemática da Bahia - Feciba como parte de sua política de Educação Científica nas escolas da rede estadual, visando disseminar uma proposta metodológica do ensino das Ciências, partindo da experimentação, da vivência da investigação científica, seus métodos e materiais, fontes de consulta e apresentação de resultados, buscando soluções para problemas contemporâneos. A Feciba tem como princípios a interdisciplinaridade, contextualização do currículo escolar e o estímulo dos estudantes ao gosto pela pesquisa e investigação como trajetória do aprendizado.

A Feciba oferta formação para os professores; realiza feiras escolares nos municípios, onde os projetos são selecionados, por um comitê científico da escola e os melhores são submetidos à Feira Estadual, que é realizada na cidade de Salvador. Na Feira Estadual, esses projetos são analisados por um Comitê Científico formado por professores das universidades baianas, das diversas áreas de conhecimento e seguem a metodologia da Febrace. Os professores e estudantes dos projetos vencedores participam da Feira Estadual com todas as despesas pagas pelo estado.

Os projetos premiados na feira estadual representam a Bahia nas férias nacionais. A Feciba é credenciada na Febrace, Ciência Jovem, Milset, Mostratec, Reunião Anual da SBPC e a Feira Nacional de Matemática. A Feciba teve financiamento do CNPq. No Quadro 1 apresenta-se o número de projetos submetidos à feira estadual, os aprovados e os vencedores nas quatro edições.

Quadro 1 - Números das edições da Feira de Ciências e Matemática da Bahia

\begin{tabular}{|l|c|c|c|c|}
\hline Projetos & $1^{\mathrm{a}}(2011)$ & $2^{\mathrm{a}}(2012)$ & $3^{\mathrm{a}}(2013)$ & $4^{\mathrm{a}}(2014)$ \\
\hline Submetidos & 84 & 90 & 351 & 416 \\
\hline Aprovados & 65 & 65 & 240 & 240 \\
\hline Premiados & 09 & 09 & 12 & 21 \\
\hline
\end{tabular}

\section{PERCURSO METODOLÓGICO}

No presente trabalho foram analisados apenas os 51 projetos vencedores nas quatro edições constantes no Quadro 2. Os resumos dos projetos vencedores das três primeiras edições se encontram disponíveis em "Feciba em Revista" (Bahia, 2014a) e, os da quarta edição, em "Feciba em Revista 2a Edição" (Bahia, 2014b).

Todos os projetos vencedores foram analisados quanto ao objeto de estudo, objetivos, procedimentos metodológicos, resultados e considerações finais. Foi criada a categoria "Envolveu Estatística" ("Sim" e "Não"), independentemente dos autores terem envolvido ou não, isto é, se pelo tema e objeto do projeto, a Estatística poderia ter sido utilizada e; a segunda categoria, foi se os autores "Utilizaram Estatística" ("Não", "Parcialmente", "Sim"). 
Quadro 2 - Projetos vencedores da Feciba quanto ao envolvimento e uso de Estatística

\begin{tabular}{|c|c|c|c|c|c|c|}
\hline \multirow[b]{2}{*}{ Edição } & \multirow[b]{2}{*}{ Área } & \multirow[b]{2}{*}{ Projeto } & \multirow{2}{*}{$\begin{array}{l}\text { Em- } \\
\text { vol- } \\
\text { veu? }\end{array}$} & \multicolumn{3}{|c|}{ Utilizaram? } \\
\hline & & & & Não & $\mathrm{P}$ & $\mathrm{S}$ \\
\hline \multirow{9}{*}{$\mathbf{1}^{\mathrm{a}}$} & \multirow{3}{*}{$\mathbf{C E}$} & Sistema de segurança para fogões contra acidentes domésticos & Sim & & & $\mathrm{X}$ \\
\hline & & $\begin{array}{l}\text { Secador Econômico para desidratação de tomates } \\
\text { reaproveitando materiais recicláveis }\end{array}$ & Sim & & $\mathrm{X}$ & \\
\hline & & Uso de material orgânico para obtenção de biogás & Sim & $\mathrm{X}$ & & \\
\hline & \multirow{3}{*}{$\mathbf{C H}$} & Geografia da fome em Jeremoabo & Sim & & & $\mathrm{X}$ \\
\hline & & Gravidez na adolescência e métodos anticoncepcionais & Sim & & & $\mathrm{X}$ \\
\hline & & Do lixo ao luxo & Não & & & \\
\hline & \multirow{3}{*}{ CB } & Casa ecológica & Não & & & \\
\hline & & Cocada de Licuri com rapadura & Sim & & $\mathrm{X}$ & \\
\hline & & Utilização da água servida no cultivo de coentro & Não & & & \\
\hline \multirow{9}{*}{$2^{\mathbf{a}}$} & \multirow{3}{*}{$\mathbf{C E}$} & $\begin{array}{l}\text { Guitar Control: uma alternativa lúdica para incentivar a prática } \\
\text { de instrumentos musicais nas crianças }\end{array}$ & Sim & & $\mathrm{X}$ & \\
\hline & & Ventilador auto-sustentável & Sim & $\mathrm{X}$ & & \\
\hline & & $\begin{array}{l}\text { Prótese de tecido para elevação do desempenho de atletas em } \\
\text { práticas desportivas com foco na velocidade }\end{array}$ & Sim & & & $\mathrm{X}$ \\
\hline & \multirow{3}{*}{$\mathbf{C H}$} & Alternativas energéticas & Sim & $\mathrm{X}$ & & \\
\hline & & $\begin{array}{l}\text { Destino dos resíduos sólidos produzidos nas empresas } \\
\text { petrolíferas do município de Catu-BA }\end{array}$ & Sim & & $\mathrm{X}$ & \\
\hline & & Cultura: desejo do povo rodelense & Sim & $\mathrm{X}$ & & \\
\hline & \multirow{3}{*}{ CB } & Canteiro Bio Séptico & Sim & $\mathrm{X}$ & & \\
\hline & & $\begin{array}{l}\text { Estudo investigativo de plantas medicinais, típicas da região } \\
\text { Nordeste do Brasil, com atividade antiparasitária em } \\
\text { camundongos }\end{array}$ & $\operatorname{Sim}$ & & & $\mathrm{X}$ \\
\hline & & Ciência e Sustentabilidade andam juntas & Não & & & \\
\hline \multirow{12}{*}{$3^{\mathbf{a}}$} & \multirow{3}{*}{$\mathbf{C E}$} & Biodigestor portátil para produção de biogás a partir de resíduos & Sim & $\mathrm{X}$ & & \\
\hline & & Caixa coletora de resíduos sólidos para bocas de lobo & Sim & & $\mathrm{X}$ & \\
\hline & & $\begin{array}{l}\text { Acidentes de trânsito: uma visão qualitativa no município de } \\
\text { Santo Estevão - BA }\end{array}$ & Sim & & & $\mathrm{X}$ \\
\hline & \multirow{3}{*}{$\mathbf{C H}$} & $\begin{array}{l}\text { A poluição do Rio Salgado, no município de Floresta Azul: } \\
\text { causas e consequências }\end{array}$ & Sim & & & $\mathrm{X}$ \\
\hline & & $\begin{array}{l}\text { A importância da acessibilidade urbana para o usuário de } \\
\text { cadeira de rodas no município de Itapetinga }\end{array}$ & $\operatorname{Sim}$ & & & $\mathrm{X}$ \\
\hline & & Ciganos: sujeitos de direitos & Sim & & & $\mathrm{X}$ \\
\hline & \multirow{3}{*}{ CB } & Irrigação e nutrição na escola pelo sistema de aquaponia & Sim & $\mathrm{X}$ & & \\
\hline & & $\begin{array}{l}\text { Protótipo para dessalinizar a água salobra do distrito de Lagoas } \\
\text { Novas, Casa Nova, BA }\end{array}$ & Não & & & \\
\hline & & Farinha da casca de abóbora & $\operatorname{Sim}$ & & $\mathrm{X}$ & \\
\hline & \multirow{3}{*}{$\mathbf{E S}$} & Produção de álcool a partir do pseudocaule da bananeira & Sim & $\mathrm{X}$ & & \\
\hline & & $\begin{array}{l}\text { Reaproveitamento do calor de fornos de panificadoras na } \\
\text { produção de energia limpa }\end{array}$ & Sim & $\mathrm{X}$ & & \\
\hline & & $\begin{array}{l}\text { Biotijolo: uma proposta de aproveitamento de materiais de } \\
\text { demolição }\end{array}$ & Sim & & & $X$ \\
\hline \multirow{4}{*}{$4^{a}$} & \multirow{3}{*}{$\mathbf{C E}$} & $\begin{array}{l}\text { Elaboração de um modelo de telha de fibrocimento adaptada } \\
\text { para um sistema de aquecedor solar integrado }\end{array}$ & Sim & & $\mathrm{X}$ & \\
\hline & & Ceativista (7 vistas) & Não & & & \\
\hline & & Capacete salva-vidas & Sim & & & $\mathrm{X}$ \\
\hline & $\mathbf{C H}$ & $\begin{array}{l}\text { Fortalecimento da identidade negra e quilombola em Antônio } \\
\text { Cardoso }\end{array}$ & Sim & & $\mathrm{X}$ & \\
\hline
\end{tabular}




\begin{tabular}{|c|c|c|c|c|c|}
\hline & Como tudo começou... Violência doméstica & Sim & $\mathrm{X}$ & & \\
\hline & Educação ambiental no Ensino Fundamental I & Sim & & $\mathrm{X}$ & \\
\hline \multirow{3}{*}{ CB } & $\begin{array}{l}\text { Aquaponia na escola como atividade inovadora de } \\
\text { aprendizagem }\end{array}$ & Não & & & \\
\hline & $\begin{array}{l}\text { Extração de amêndoa do caroço da manga na produção de } \\
\text { gêneros alimentícios }\end{array}$ & Sim & $\mathrm{X}$ & & \\
\hline & $\begin{array}{l}\text { Controle alternativo de cochonilhas em palmas forrageiras na } \\
\text { localidade de Serra Branca, Casa Nova-BA }\end{array}$ & Sim & $\mathrm{X}$ & & \\
\hline \multirow{3}{*}{$\mathbf{E S}$} & $\begin{array}{l}\text { Conversor eletromagnético: utilizando forças magnéticas para } \\
\text { obtenção de energia elétrica }\end{array}$ & Sim & $\mathrm{X}$ & & \\
\hline & $\begin{array}{l}\text { Assistência técnica rural - sustentabilidade para a agricultura } \\
\text { familiar do município de Itororó-BA }\end{array}$ & Sim & $\mathrm{X}$ & & \\
\hline & Eco-banho & Sim & & & $\mathrm{X}$ \\
\hline \multirow{3}{*}{ DC } & $\begin{array}{l}\text { Sistema de segurança do bebê ou portador de necessidades } \\
\text { especiais }\end{array}$ & Sim & & $\mathrm{X}$ & \\
\hline & Robô de tele presença controlado & Não & & & \\
\hline & Pratinho de planta anti-dengue & Sim & & $\mathrm{X}$ & \\
\hline \multirow{3}{*}{ MF } & Calculando o IMC utilizando a planilha Calc & Sim & & & $\bar{X}$ \\
\hline & $\begin{array}{l}\text { Calcular, efetuar, encontrar o X da questão... isso não me torna } \\
\text { racional, e sim, mecânico }\end{array}$ & Não & & & \\
\hline & Cacau: fonte de renda e aprendizagem & Sim & & & $\mathrm{X}$ \\
\hline \multirow{3}{*}{ MM } & Xadrez - Ferramenta pedagógica na Matemática & Sim & & & $\mathrm{X}$ \\
\hline & $\begin{array}{l}\text { O uso de jogos para o desenvolvimento do raciocínio lógico } \\
\text { matemático }\end{array}$ & Não & & & \\
\hline & O homem que calculava - O jogo & Não & & & \\
\hline
\end{tabular}

Legenda: Utilizaram?: Não, $\mathrm{P}=$ Parcialmente, $\mathrm{S}=$ Satisfatoriamente.

Área: $\mathrm{CE}=$ Ciências Exatas, $\mathrm{CB}=$ Ciências Biológicas e da Saúde; $\mathrm{CH}=$ Ciências Humanas; ES=Energia e Sustentabilidade: DC=Divulgação Científica; $\mathrm{MF}=$ Matemática Ensino Fundamental e MM=Matemática Ensino Médio.

Analisando os 51 projetos vencedores verifica-se que 40 (78,4\%) envolviam algum tipo de conteúdo de estatística. Contudo, desses 14 (35\%) não utilizaram nenhum conteúdo, 11 (27,5\%), utilizaram parcialmente (P) e 15 (37,5\%) utilizaram satisfatoriamente (S).

A maioria dos projetos que não utilizou Estatística (Não) poderia ter usado pelo menos a "leitura de informações estatísticas" para embasar a argumentação do trabalho e, em alguns casos, poderia ter optado por fazer algum tipo de levantamento estatístico para conduzir a investigação, como por exemplo, o projeto "Assistência técnica rural - sustentabilidade para a agricultura familiar do município de Itororó-BA", cujo foco foi acompanhar e assistir aos produtores. Neste caso, a utilização da Estatística poderia subsidiar, por exemplo, o mapeamento das principais dificuldades encontradas por essa comunidade.

Os projetos que utilizaram parcialmente Estatística $(\mathrm{P})$ o fizeram para introduzir o problema, trazendo para a discussão informações estatísticas disponíveis na literatura existente (uso de dados secundários, leitura de dados), mas os projetos pelo seu delineamento comportavam o uso de Estatística que poderiam enriquecer o trabalho. Um exemplo é o projeto "Secador Econômico para desidratação de tomates reaproveitando materiais recicláveis", no qual os autores delinearam um artefato para desidratar os tomates. Neste caso, os autores poderiam ter realizado vários ensaios, tomando medidas tais como o tempo, o peso dos tomates frescos, dos desidratados, a temperatura, entre outras, para assim calcular o tempo médio, o peso médio etc.

Já os projetos que utilizaram satisfatoriamente (S), exploraram o potencial da Estatística, como é o caso do projeto "Geografia da fome em Jeremoabo", onde os autores inspirados no livro de Josué de Castro realizaram uma investigação estatística, percorrendo todas as fases da investigação científica. Apresentado e premiado na Febrace, este projeto foi orientado por professores de Geografia. O projeto identifica os problemas que contribuem para a manutenção de pessoas abaixo da linha de pobreza e seu impacto no desenvolvimento educacional. 


\section{REFLEXÕES E CONSIDERAÇÕES}

A Feciba tem tido a capacidade de mobilizar a escola pública na busca de espaços de realização de projetos de investigação, em geral, interdisciplinares, focando temas de relevância social, propiciando o desenvolvimento do trabalho colaborativo, instigando os estudantes e professores a tomarem uma atitude ativa e propositiva, nos marcos do Letramento Estatístico proposto por Gal (2002).

Nesse contexto, observa-se que são os professores de outras áreas do conhecimento que encabeçam a orientação dos trabalhos e que raramente os professores de Matemática fazem parte da equipe. Outra constatação é que conceitos e procedimentos estatísticos que não são recomendados na Educação Básica, fazem parte do cotidiano dos cidadãos tais como nível de confiança, margem de erro, amostragem, tipo de amostra dentre outros. Contata-se, também, com raras exceções, que os projetos que utilizam Estatística privilegiam as variáveis qualitativas o que limita as potencialidades do uso da Estatística.

Os resultados da Feciba mostram que é possível um trabalho interdisciplinar na escola pública e que a Estatística pode potencializar a leitura, a modelação dos fenômenos naturais e sociais que permeiam o cotidiano escolar e que podem contribuir na formação de cidadãos críticos, no sentido de compreender e questionar as informações a eles expostas e formular e realizar investigações que lhes permitam a construção de sua autonomia.

\section{REFERÊNCIAS}

Bahia (2014a). Secretaria da Educação. Feciba em Revista. Disponível em: $<$ http://issuu.com/iat1/docs/feciba revista final $>$, acesso em 15 mar. 2015.

Bahia (2014b). Secretaria da Educação. Feciba em Revista, 2a Edição. Disponível em:

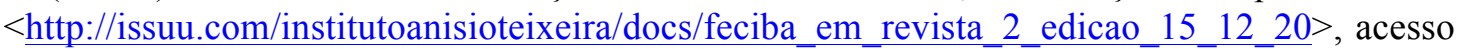
em 15 mar. 2015.

Batanero, C. (2001). Didáctica de la Estadística. Granada: Disponível em: $<$ http://www.ugr.es/ batanero/publicaciones\%20index.htm>, acesso em 15 mar. 2015.

Brasil (1996). Ministério da Educação. Lei de Diretrizes e Bases da Educação Nacional - LDB. Lei $\mathrm{n}^{\mathrm{o}}$ 9.394, de 20 de dezembro de 1996.

Brasil (1997). Ministério da Educação. Secretaria de Educação Fundamental. Parâmetros Curriculares Nacionais: Matemática. Brasília.

Brasil (1998). Ministério da Educação. Secretaria de Educação Fundamental. Parâmetros Curriculares Nacionais: Matemática. Brasília.

Brasil (2002), Ministério da Educação. Secretaria de Educação Média e Tecnológica. PCN Ensino Médio: Orientações Educacionais complementares aos Parâmetros Curriculares Nacionais Ciências da Natureza, Matemática e suas Tecnologias. Brasília.

Brasil (2014a), Ministério da Educação. Plano Nacional de Educação 2014-2024, disponível em: $<$ http://pne.mec.gov.br/>, acesso em 15 mar. 2015.

Brasil (2014b), Ministério da Educação. Base Nacional Comum da Educação, disponível em: $<$ http://basenacionalcomum.org.br/>, acesso em 15 mar. 2015.

Cazorla, I. (2006). Teaching statistics in Brazil. In A. Rossman, \& B. Chance, (Eds.), Proceedings of Seventh International Conference Teaching Statistics. International Association for Statistical Education, Salvador, Brazil [CD-ROM].

Cazorla, I., \& Castro, F. C. (2008). Papel da Estatística na leitura do mundo: o letramento estatístico. Publicatio UEPG: Ciências Humanas, Ciências Sociais Aplicadas, Linguística, Letras e Artes, 16 (1), 45-53.

Cazorla, I. \& Santana, E. (Org.). (2010). Do Tratamento da Informação ao Letramento Estatístico. Itabuna: Via Litterarum.

Cazorla, I., Kataoka, V. Y., \& Silva, C. B. (2010). Trajetórias e Perspectivas da Educação Estatística no Brasil: um olhar a partir do GT12. In: Lopes, C. E.; Coutinho, C.; Almouloud, S. (Org.) Estudos e Reflexões em Educação Estatística. São Paulo: Mercado das Letras.

Gal, I. (2002). Adult's Statistical Literacy: meanings, components, responsibilities. International Statistical Review, 70(1), $1-25$.

Viali, L. (2008). Ensino de Estatística e Probabilidade nos Cursos de Licenciatura em Matemática. SINAPE (Simpósio Nacional de Probabilidade e Estatística). Estância de São Pedro (SP). 